that, without the additional buyers and bigger market, NASA cannot afford to pay for the upkeep of the launch-pad infrastructure that the Air Force had paid for. "NASA can't go it alone," says Wrobel.

Wrobel says that most imminent mission launches, such as the 2011 launch of the heavy Mars Science Laboratory and Juno, a mission to Jupiter, needed the extra thrust of an Atlas V anyway. But if NASA officials are forced to buy more Atlas Vs in the future, they will be paying extra for unused launch capacity. "The more that the launch vehicle costs, the less science mission you get for your money. Or fewer missions," says Alan Stern, a planetary scientist at Southwest Research Institute in Boulder, NASA's former science chief and an advocate of commercial space flight.

Several missions could take advantage of Falcon 9's leaner launch capability and lower price of about $\$ 50$ million per launch. These include the Soil Moisture Active and Passive mission, an Earth-observing satellite due for launch in 2015; the International Lunar Network, a system of landers

$\begin{array}{ll}\text { "The more the } & \begin{array}{l}\text { designed to meas- } \\ \text { ure the Moon's seis- } \\ \text { mic activity, among } \\ \text { other things; and } \\ \text { launch vehicle }\end{array} \\ \begin{array}{ll}\text { costs, the less } & \text { modest-sized } \\ \text { science mission } & \text { astrophysics and } \\ \text { you get for youretary-science }\end{array} \\ \text { money." } & \begin{array}{l}\text { missions that would } \\ \text { launch in 2016. }\end{array}\end{array}$

All bets are not riding on the Falcon 9, however, which will launch from Cape Canaveral, Florida, and carry a prototype of its cargo capsule Dragon. Orbital Sciences, headquartered in Dulles, Virginia, and another winner of ISS cargo-transport money, is developing the Taurus II, another medium-sized launcher that is scheduled for first test flights in 2011 (see Mid-sized rockets need a boost). For the planned 2012 launch of the Lunar Atmosphere and Dust Environment Explorer (LADEE), Orbital is putting together stockpiled ballistic missiles into the Minotaur 5, which costs less than $\$ 50$ million and is just the right size for the small Moon mission.

If Falcon 9's test launch is successful, it should be carrying cargo to the ISS within a few months. But scientists will have to wait a while longer - before a new rocket can carry a scientific payload, NASA requires three successful launches and a technical certification that takes about three years. NASA hopes to certify Falcon 9 or one of its competitors by the end of 2013 .

Eric Hand

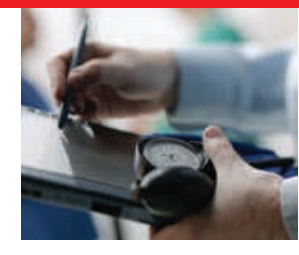

DOCTORS SEEK REFORM Cumbersome rules for UK clinical trials are driving research overseas. go.nature.com/q2q7BT

\title{
Neglected diseases fund touted
}

Despite decades of research into drugs and vaccines for neglected diseases such as tuberculosis and dengue fever, few products have made it through clinical development and into the hands of the millions who desperately need them.

One of the biggest hurdles is the sheer expense of running clinical trials, compared with the small profits that commercial companies can expect to make from treatments for diseases that disproportionately affect poor and marginalized populations.

This week, alongside the World Health Organization's (WHO's) annual assembly of health ministers in Geneva, Switzerland, a consortium of industry and nongovernmental organizations proposed a scheme to help address the problem: a global fund that would channel billions of dollars a year into product development.

Plans for the fund were put forward by the International AIDS Vaccine Initiative (IAVI), the pharmaceutical giant Novartis and the George Institute for International Health in Sydney, Australia. It would channel money from donors towards product-development partnerships (PDPs) - collaborative efforts between research agencies, donors and biotech and pharmaceutical companies to develop drugs, diagnostics and vaccines for the developing world. The fund aims to encourage contributions from donors who lack the resources or expertise to assess the quality and progress of the various PDP offerings.

Dozens of not-for-profit PDPs, including the IAVI, the Medicines for Malaria Venture, the Global Alliance for TB Drug Development, and the Drugs for Neglected Diseases initiative (DNDi), have been set up during the past 15 years. Their aim is to bridge the gap between basic research and product development, and to prevent promising research leads for neglected diseases from languishing on the shelf. They are run like businesses, but are supported by donor funding, and have generous intellectual-property rules to make any products affordable to poor countries, allowing generic manufacturers to make cheap versions freely.

PDPs have become an attractive choice for neglected-disease donors - of the estimated US $\$ 3$ billion spent on such research in 2008, about one-fifth was channelled through PDPs. Although only 13 of more than 1,000 drugs developed between 1975 and 1997 were for neglected diseases, PDPs created over the past decade already have 143 candidate products in development, and have rolled out 11 products for malaria, leishmaniasis and meningitis.

But many PDPs need a fresh cash injection as more of their product candidates begin to enter clinical development, the most expensive phase of drug and vaccine discovery. Without substantial new funding, projects will stall and waste much of the earlier development work and investments, says Paul Herrling, head of the Novartis Institutes for Developing World Medical Research.

The proposed PDP+ Fund would seek to raise funds from governments and other donors, and through bond financing and innovative taxation schemes. It would act as a one-stop-shop for donors, coordinating funding of projects to many different PDPs.

"Do we need a super-PDP fund? Without a doubt," says Jean-François Alesandrini, a spokesman for the DNDi. However, he cautions that the details of the scheme still need fleshing out, particularly on the issue of attracting new donor funding. Many questions remain as to how the PDP+ Fund would work, adds Alesandrini, in particular its governance structure, and how its expert committees would choose which projects to fund.

Herrling says that informal discussions he has had with donors, including the US government, have been positive, as have those with other companies and groups that will be invited to come on board.

The PDP+Fund resembles the idea for a global research fund that was floated by a WHO expert panel in 2002 to complement the multibillion-dollar Global Fund to Fight AIDS, Tuberculosis and Malaria. This was created in the same year, but funds only disease-control measures, not research. The global research fund proposal never gained traction because governments considered it a risky venture, says Mary Moran, director of health policy at the George Institute. The difference now, says Herrling, is that PDPs are widely recognized to produce drug leads. "We have a state-of-the-art pipeline that now needs investment to take forward," he says.

Declan Butler 